\title{
Phylogenetic relationships among extinct and extant turtles: the position of Pleurodira and the effects of the fossils on rooting crown-group turtles
}

\author{
Juliana Sterli ${ }^{1,2}$ \\ ${ }^{1}$ CONICET - Museo Paleontológico Egidio Feruglio, Av. Fontana 140, 9100 Trelew, Chubut, Argentina \\ ${ }^{2}$ E-mail: jsterli@mef.org.ar
}

Key words: molecules, morphology, phylogeny, Testudinata, Testudines

\begin{abstract}
The origin and evolution of the crown-group of turtles (Cryptodira + Pleurodira) is one of the most interesting topics in turtle evolution, second perhaps only to the phylogenetic position of turtles among amniotes. The present contribution focuses on the former problem, exploring the phylogenetic relationships of extant and extinct turtles based on the most comprehensive phylogenetic dataset of morphological and molecular data analyzed to date. Parsimony analyses were conducted for different partitions of data (molecular and morphological) and for the combined dataset. In the present analysis, separate analyses of the molecular data always retrieve Pleurodira allied to Trionychia. Separate analysis of the morphological dataset, by contrast, depicts a more traditional arrangement of taxa, with Pleurodira as the sister group of Cryptodira, being Chelonioidea the most basal cryptodiran clade. The simultaneous analysis of all available data retrieves all major extant clades as monophyletic, except for Cryptodira given that Pleurodira is retrieved as the sister group of Trionychia. The paraphyly of Cryptodira is an unorthodox result, and is mainly caused by the combination of two factors. First, the molecular signal allies Pleurodira and Trionychia. Second, the morphological data with extinct taxa locates the position of the root of crown-group Testudines in the branch leading to Chelonioidea. This study highlights major but poorly explored topics of turtle evolution: the alternate position of Pleurodira and the root of crown turtles. The diversification of crown turtles is characterized by the presence of long external branches and short internal branches (with low support for the internal nodes separating the major clades of crown turtles), suggesting a rapid radiation of this clade. This rapid radiation is also supported by the fossil record, because soon after the appearance of the oldest crown-group turtles (Middle-Late Jurassic of Asia) the number and diversity of turtles increases remarkably. This evolutionary scenario of a rapid diversification of modern turtles into the major modern lineages is likely the reason for the difficulty in determining the interrelationships and the position of the root of crown-group turtles.
\end{abstract}

\section{Contents}

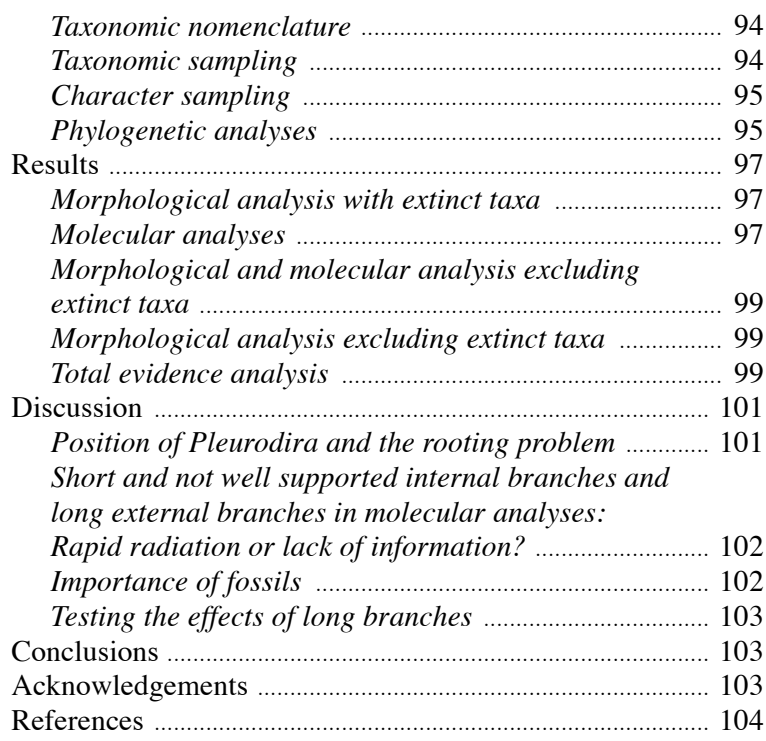

\section{Introduction}

Testudinata (sensu Joyce et al., 2004) is a highly enigmatic clade of amniotes that is primarily characterized by the development of a bony shell that encloses the pectoral girdles within the rib cage (Burke, 1989). Although testudinates are abundant in the fossil record, earliest forms remain relatively rare thus resulting in significant morphological gaps. As a consequence, both the origin of turtles and the phylogenetic relationships of the turtle crown-group are issues that remain controversial to date. In some way both issues are related. In fact, the rooting of crown-group turtles is difficult because a long branch separates them from their extant sister-group (e.g. archosaurs, lepidosaurs).

The question of the origin of turtles is a subject that has received much attention during the last decades through the development of several phylogenetic 
analyses based mainly in morphological data. Among basal amniotes, turtles have been hypothesized to be the sister group of procolophonoids (Reisz and Laurin, 1991; Laurin and Reisz, 1995), pareiasaurs (Lee, 1993, 1995, 1996, 1997), and captorhinomorphs (Gauthier et al., 1988). Similarly, within the derived reptilian clade Diapsida, turtles have been placed as sister to lepidosaurs (Müller, 2003, 2004; Hill, 2005) and sauropterigyans (Rieppel and deBraga, 1996; deBraga and Rieppel, 1997; Rieppel and Reisz, 1999).

On the other hand, a large number of phylogenetic studies have been published on the evolution of the clade of turtles based on both morphological and molecular data over the course of the last fifteen years. The pioneer phylogenetic analysis of turtle evolution based on morphological data was published by Gaffney (1975) and it provided the basis of a series of further studies (see Gaffney et al., 2007 and references therein). These studies established the interrelationships of the major clades of extinct and extant turtles and retrieved a major split in crown-group turtles, separating Cryptodira from Pleurodira. All published analyses of morphological data have since agreed with this basic result, although the position of several extinct groups is still debated. One group of studies retrieved all extinct turtles, except for three taxa (Proganochelys quenstedti Baur 1887, Palaeochersis talampayensis Rougier, de la Fuente and Arcucci 1995, and Australochelys africanus Gaffney and Kitching 1994), as belonging to either Cryptodira or Pleurodira based on morphological evidence (e.g. Gaffney, 1975; Gaffney and Meylan, 1988; Gaffney, 1996; Hirayama et al., 2000; Gaffney et al., 2007; Sterli et al., 2007). Another group of morphology-based studies place additional extinct taxa outside the crown-group turtles (Rougier et al., 1995; Joyce, 2007; Danilov and Parham, 2006, 2008; Sterli, 2008; Anquetin et al., 2009).

Molecular analyses, finally, have provided a wealth of new information on the interrelationships among the major clades of extant turtles (Fujita et al., 2004; Krenz et al., 2005; Near et al., 2005; Parham et al., 2006; Iverson et al., 2007; Thomson and Shaffer, 2010). The genes sequenced in these studies include mitochondrial genes (cytochrome b, 12S RNA) and nuclear genes (RAG-1, intron fingerprint protein R35) and were analyzed with parsimony and statistical (maximum likelihood and Bayesian analysis) methods. These molecular studies have shed much light and new data on the affinities of some clades of extant turtles (compared to the results obtained with morphological data) retrieving as a major novel result the basal position of Trionychia as being the sister clade of all remaining extant cryptodiran turtles. Combined analysis of major turtle groups based on morphological and molecular data, however, are still scarce in the literature. Shaffer et al. (1997) is the only study that has used this approach so far. They analyzed 30 species (23 extant and 7 extinct) using 2 mitochondrial genes (cytochrome $b$ and 12S RNA) and 115 morphological characters. In the total evidence analysis and in the morphological analyses with some or with all the extinct taxa included, Shaffer et al. (1997) retrieved Pleurodira and Cryptodira as monophyletic groups and Proganochelys quenstedti being the only extinct taxon located outside crown-group turtles.

The main objectives of the present paper are, first to explore the interrelationships of the major groups of turtles including a much larger sample of extinct and extant taxa and morphological and molecular characters than in previous analyses. Second, analyze the phylogenetic signal provided by different types of data, and third evaluate the impact of extinct taxa on the phylogenetic results. This contribution specifically focuses on the conflicting phylogenetic signal on the position of Pleurodira and the impact of extinct taxa on determining the placement of the root of crown-group turtles.

\section{Material and methods}

\section{Taxonomic nomenclature}

Suprageneric taxon names are used as defined by Joyce et al. (2004). Testudines refers to the "crown clade arising from the last common ancestor of Chelonia (orig. Testudo) mydas and Chelus (orig. Testudo) fimbriatus' and all their descendents (Joyce et al., 2004). Testudinata refers to the 'apomorphy-based version of Testu$d o$ ' and includes crown group Testudines plus all stem taxa 'with a complete turtle shell that is homologous with the shell present in Chelonia (orig. Testudo) mydas' (Joyce et al., 2004). Throughout this paper the term 'clade' or 'group' refer to the terms 'subfamily', 'family', or 'superfamily' of the rank-based nomenclature. The terminology for unrooted trees follows Wilkinson et al. (2007), where 'adjacent group' refers to the 'unrooted analogue of sister group'.

\section{Taxonomic sampling}

The morphological matrix is based on the phylogenetic analysis of Sterli (2008), however, 8 taxa were 
added (see on-line supplementary information -SI- 1 and 2) and the dataset, consequently, includes 51 extinct and 27 extant species of turtles and four outgroup taxa. This taxon sampling scheme almost triples the number of taxa included in the only combined analysis of molecular and morphological data performed to date (Shaffer et al., 1997). This difference is mainly due to the inclusion of a large number of extinct taxa that were absent in the mentioned study. The taxon sampling includes representatives of the two major groups of Pleurodira (Chelidae and Pelomedusoides) and the major clades of Cryptodira (Trionychia [Carettochelyidae + Trionychidae], Kinosternoidea [Kinosternidae + Dermatemydidae], Chelonioidea [Cheloniidae + Dermochelyidae], Platysternidae, Chelydridae, Testudinidae, Geoemydidae, and Emydidae). Furthermore many stem Testudines (e.g. Proganochelys quenstedti, Palaeochersis talampayensis, Australochelys africanus), as well as some stem representatives of the major subclades of crown group turtles [e.g. Protochelydra zangerli Erickson 1973, Mongolemys elegans Khozatskii and Mlynarski 1971, Hoplochelys crassa (Cope 1888), Adocus beatus (Leidy 1865)], were included.

The selection of outgroup taxa is related to the main hypotheses of turtle's sister groups based on morphological data. Simosaurus gaillardoti (Meyer, 1842) was chosen because Rieppel and deBraga (1996), deBraga and Rieppel (1997) and Rieppel and Reisz (1999) argued that turtles were the sister group of sauropterygians. Sphenodon punctatus Gray 1842 was chosen because Müller $(2003,2004)$ and Hill (2005) suggested lepidosauromorphs were the sister clade of turtles. Owenetta kitchingorum Reisz and Scott 2002 was chosen because Reisz and Laurin (1991) and Laurin and Reisz (1995) suggested procolophonoids as the sister group of turtles, while Anthodon serrarius Owen 1876 was chosen because Lee $(1993,1995)$ supported a sister group relationship between pareiasaurs and turtles.

\section{Character sampling}

The central aim of this contribution is the analysis of the available morphological and molecular data frequently used to explore the phylogenetic relationships of extant and extinct turtles. The morphological matrix includes 152 characters and is based on Sterli (2008), but three new characters were added and certain changes were performed in the scorings of some taxa (see SI 1). Molecular data includes all sequences that have been used for phylogenetic analyses of turtle evolution and include three mitochondrial (12S RNA, 16S RNA, and cytochrome b) and two nuclear genes (RAG-1 and the intron of the fingerprint protein 35 [R35]). Nuclear genes are supposed not to be as saturated as mitochondrial genes and, consequently, they may be more adequate to analyze deeper cladogenesis (Engstrom et al., 2004). However, only two nuclear genes (RAG-1 and R35) have been used to explore the phylogenetic relationships among the major clades of turtles and are sequenced in at least one species per major clades (Fujita et al., 2004; Krenz et al., 2005; Near et al., 2005). Several phylogenetic analyses also included mitochondrial genes (Shaffer et al., 1997; Krenz et al., 2005) and therefore these are also included here (12S RNA, 16S RNA, and the cytochrome b). All the sequences were downloaded from GenBank (http://www.ncbi.nlm.nih.gov/) and their accession numbers are listed in SI 3.

\section{Phylogenetic analyses}

The phylogenetic analyses were performed using the program TNT (Goloboff et al., 2008a, b). For the parsimony analysis all characters were treated as unordered and equally weighted. The transformation cost between transitions and transversions was considered equal and gaps were considered as a fifth state to preserve its phylogenetic information (Giribet and Wheeler, 1999). A total evidence analysis was conducted combining the morphological and molecular information, as well as several partitioned analyses to analyze the phylogenetic signal of each dataset (e.g. molecular vs. morphological, extant vs. extinct taxa, comparisons of the five genes). Given the presence of highly divergent taxa, denoted by the presence of long branches, several molecular analyses were run excluding these terminal taxa (e.g. Trionychia and Pleurodira). This procedure, known as long branch extraction (LBE; Siddall and Whiting, 1999; Pol and Siddall, 2001), aims to test the influence of long branches on the resultant topologies (e.g. long branch attraction).

The heuristic tree search consisted of a thousand replicates of Wagner trees (using random addition sequences) followed by Tree Bisection Reconnection (TBR) branch swapping (saving 10 trees per replication). All the most parsimonious trees found in the replicates were subject to a final round of TBR.

Branch support was calculated using Jackknife resampling (Lanyon, 1985) as incorporated in TNT 
A

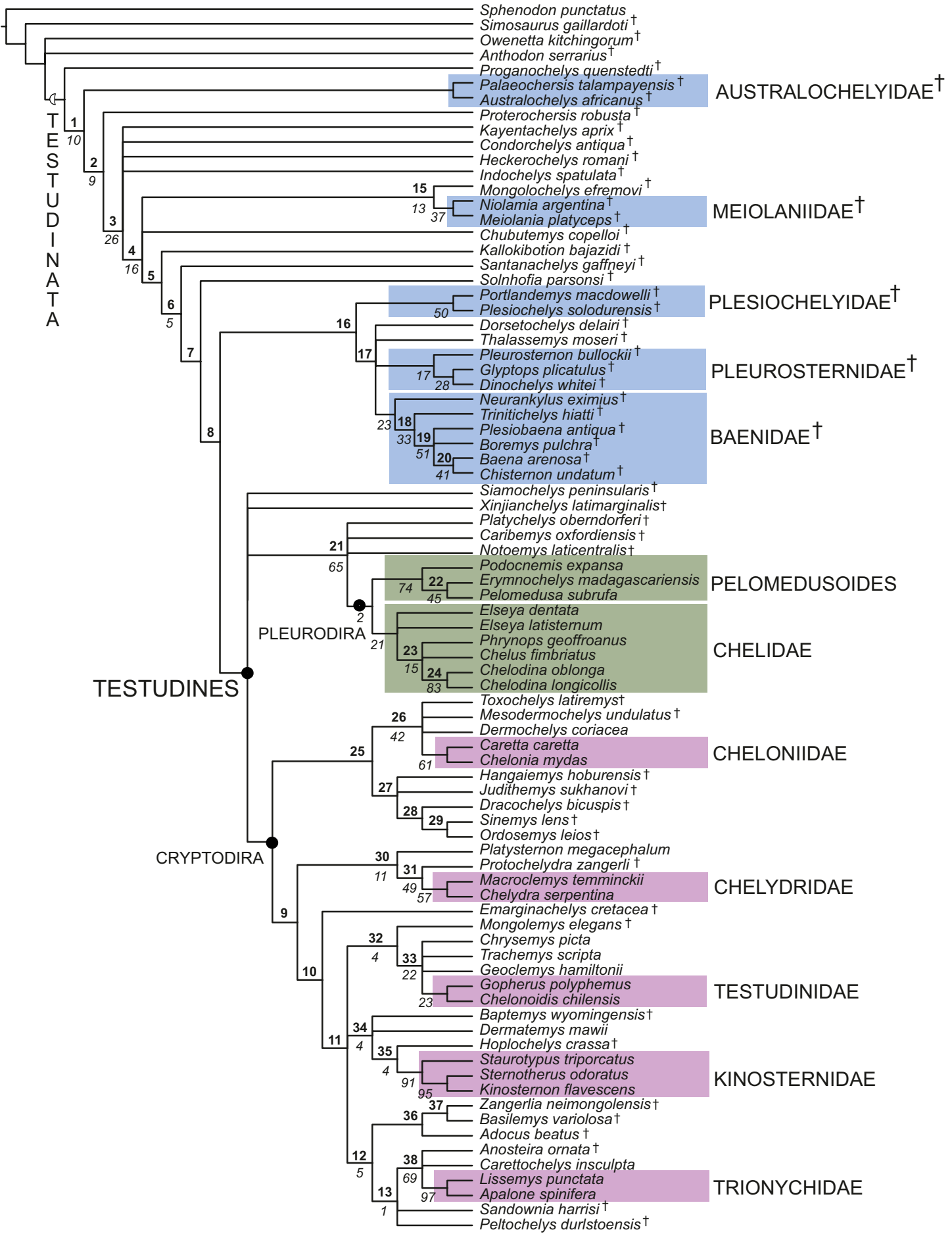

Fig. 1. Morphological phylogenetic analysis of extinct and extant taxa. Strict consensus tree of 19440 MPTs of 479 steps each. Numbers in bold above the nodes represent node number. Numbers in italics under the nodes represent GC Jackknife values (for Group present/ Contradicted; difference between the frequency in which a given group is retrieved in the jackknife replicates and the most frequent contradictory group). Light blue: extinct recognized clades. Pink: Cryptodiran clades. Green: Pleurodiran clades. 
(see Goloboff et al., 2003). One thousand jackknife replicates were performed conducting a heuristic tree search consisting of 10 replicates of Wagner trees (with random addition sequences) followed by TBR (saving 10 trees per replicate). The results shown are difference in frequencies GC (for Group present/Contradicted) developed by Goloboff et al. (2003). The difference in frequencies GC was chosen because it is calculated as the difference between the frequency in which a given group is retrieved in the jackknife replicates and the most frequent contradictory group (Goloboff et al., 2003). Absolute frequencies (the usual method of counting frequencies in jackknife or bootstrap analysis) do not distinguish between a group with a frequency of 0.6 that is never contradicted, and a group with a frequency of 0.6 that is contradicted with a frequency of 0.4 . GC frequencies distinguish these two cases, giving lower support values to the second type of groups (Goloboff et al., 2003).

\section{Results}

The Clustal alignment (Thompson et al., 1997; for more information on alignment parameters see SI 1) produced the following data matrices: 12S RNA (24 taxa and 350 nucleotides sites), 16S RNA (20 taxa and 489 characters), cytochrome b (22 taxa and 892 characters), RAG-1 (17 taxa and 2790 characters), and the intron of the fingerprint protein 35 (18 taxa and 990 characters). See SI 4 through 8 for information on these datasets. The complete data set including molecular and morphological data was formed of 83 taxa and 5663 characters. Of the molecular characters, $23 \%$ are parsimony informative.

\section{Morphological analysis with extinct taxa}

The cladistic analysis of extinct and extant taxa based exclusively on morphological data resulted in 19,440 most parsimonious trees (MPTs) of 479 steps. The strict consensus is shown in Fig. 1 (common synapomorphies are listed in SI 9). In these trees, many groups of extinct turtles are placed along the stem of crown group Testudines. These include the most ancient turtles from the Late Triassic (Proganochelys quenstedti, Proterochersis robusta Fraas 1913, Palaeochersis talampayensis), Kayentachelys aprix Gaffney, Hutchison, Jenkins, and Meeker 1987, Condorchelys antiqua Sterli 2008, Heckerochelys romani Sukhanov 2006, and the extinct groups Meiolaniidae, Plesio- chelyidae, Pleurosternidae, and Baenidae. The MPTs therefore resemble in the position of these extinct taxa emerging alternative hypotheses on turtle evolution (Joyce, 2007), including the morphological study of Sterli (2008). Pleurodira and Cryptodira are closely related and form a basal polytomy whith Xinjiangchelys latimarginalis (Young and Chow 1953) and Siamochelys peninsularis Tong, Buffetaut, and Suteethorn 2004, due to alternative positions of the later two species. All main groups of crown-group turtles recovered as monophyletic. Pleurodira splits in Chelidae and Pelomedusoides, and within Cryptodira, the taxa Trionychidae, Kinosternidae, Testudinidae, Chelydridae, Cheloniidae, and Emydidae, with their basal members, were recovered as monophyletic. In this analysis Cheloniidae and related taxa (plus 'sinemyds' and 'macrobaenids') form the sister group of the remaining crown Cryptodira. The position of Chelonioidea as the sister group of the remaining extant Cryptodira was also suggested by Brinkman and $\mathrm{Wu}$ (1999), Joyce (2007), and Werneburg and SánchezVillagra (2009). Although some differences exist, this result is similar to those found by Joyce (2007), Danilov and Parham (2008), and Sterli (2008). In this analysis the root of crown-group Testudines is located between Cryptodira and Pleurodira (Fig. 5).

The following partitioned results are shown as unrooted trees to facilitate the comparison among partitions.

\section{Molecular analyses}

Only one MPT of 5483 steps (Fig. 2A) was obtained analyzing the concatenated data matrix of molecular data including five genes (12S RNA, 16S RNA, cytochrome b, RAG-1, and intron of the fingerprint protein R35). The molecular partition retrieved as monophyletic the major groups of crown turtles: Chelidae and Pelomedusoides for Pleurodira and Trionychia, Kinosternoidea, Testudinidae + Geoemydidae, Chelydridae, Chelonioidea, and Emydidae for Cryptodira. Almost all of these clades are well supported by Jackknife values (>80\%); however, no Jackknife values were recovered for the internal branches, except for the branch dividing Pleurodira and Cryptodira. The analysis depicts Kinosternoidea and Trionychia as the successive adjacent groups of Pleurodira showing that the molecular signal is responsible for the close affinities of Pleurodira and Trionychia in the total evidence analysis (see below).

The separate analyses of each of the analyzed genes are summarized in Fig. 2B-F. Given that the 
A

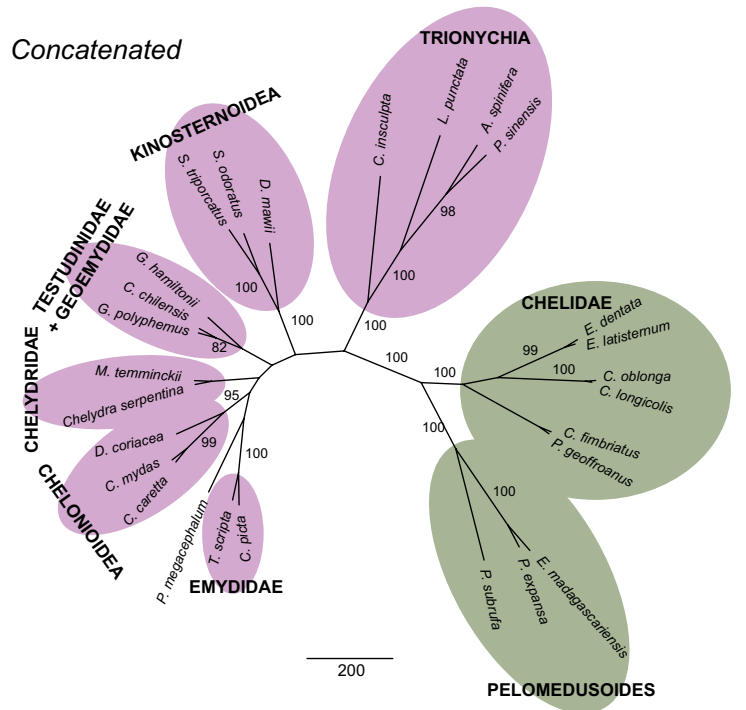

C

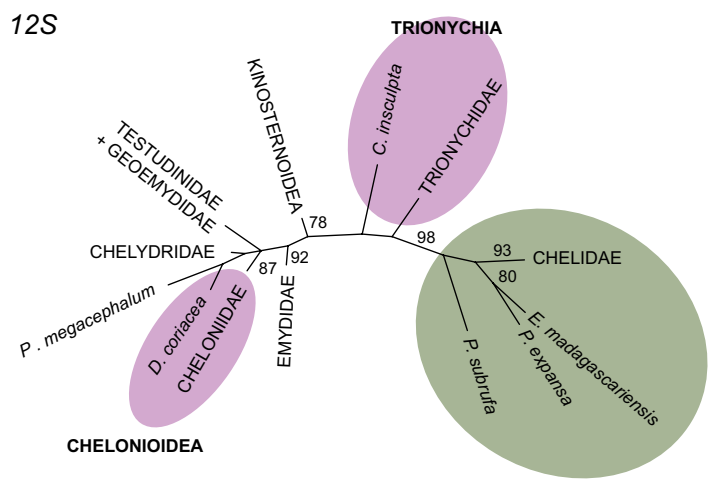

$\mathrm{E}$

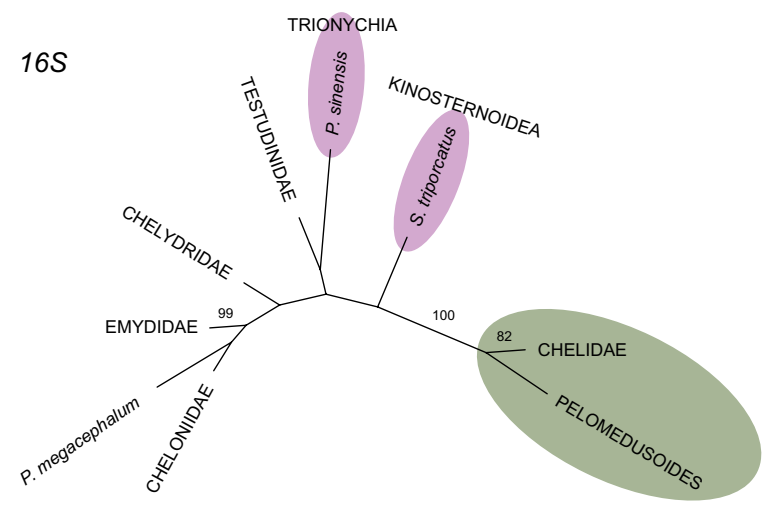

B

Cytochrome $b$

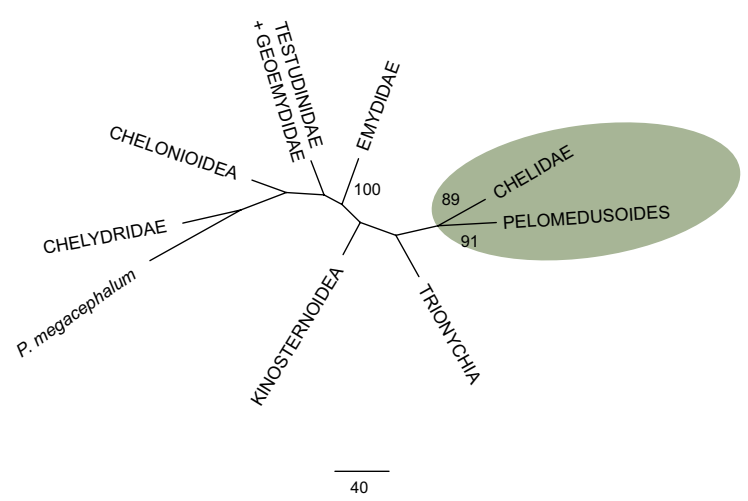

D

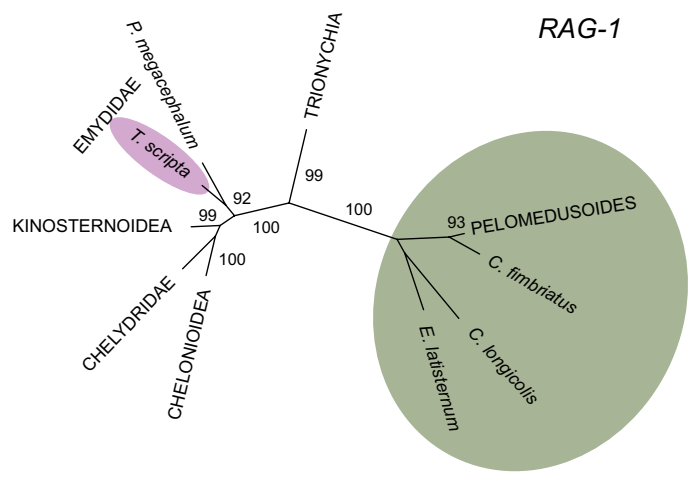

$\mathrm{F}$

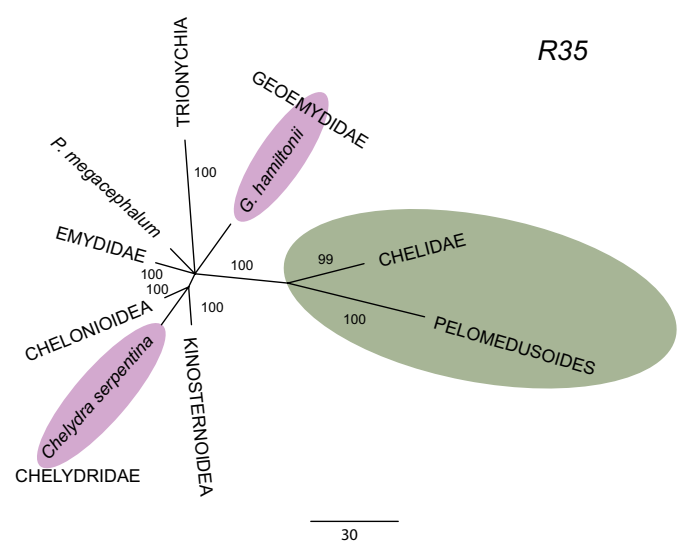

Fig. 2.A) Molecular phylogenetic analysis of the 5 sequences (12S RNA, 16S RNA, cytochrome b, RAG-1, and intron of the fingerprint protein 35). Only one MPT of 5483 steps were recovered. B) Molecular analysis of cytochrome b sequence. Only one MPT of 2061 steps was recovered. C) Molecular analysis of 12S RNA sequence. Strict consensus of two MPTs of 644 steps each. D) Molecular analysis of the RAG-1 sequence. Only one MPT of 927 steps was recovered. E) Molecular analysis of 16S RNA sequence. Only one MPT was found of 838 steps. F) Molecular analysis of the intron of the fingerprint protein 35. Strict consensus of two MPTs of 972 steps each. Jackknife values below 70 are not shown. Pink: Cryptodiran clades. Green: Pleurodiran clades. 
most striking differences in the results of the present analyses are the affinities of Pleurodira, one of the two major groups of living turtles, I will focus on the adjacent groups of Pleurodira. The phylogenetic analyses of the sequences of cytochrome b (one MPT of 2061 steps; Fig. 2B), 12S RNA (two MPTs of 644 steps; strict consensus in Fig. 2C), and RAG-1 (one MPT of 927 steps; Fig. 2D) show that the adjacent group of Pleurodira is Trionychia, followed by the group Kinosternoidea. The results of these three genes generally resemble the topology obtained with that of the concatenated data matrix of all the molecules (Fig. 2A), except for the position of Emydidae that is closer to Kinosternoidea and/or Trionychia (Fig. 2B-D) than in the analysis of the five genes.

On the other hand, the topologies obtained in the analyses of the sequences of 16S RNA and the intron fingerprint protein R35 are slightly different. The result of the sequence 16S RNA (one MPT of 838 steps) shows Kinosternoidea as the adjacent group of Pleurodira, however, the Jackknife values are very low (Fig. 2E). The later result could be affected by the scarce sequence information on trionychians and kinosternoids that are represented in this analysis by only one species each. The result obtained for analysis of the intron of the fingerprint protein 35 (two MPTs of 972 steps) shows that the interrelationships among Cryptodira are not resolved and therefore there is no clear signal to test which of the cryptodiran groups is closer to Pleurodira (Fig. 2F)

\section{Morphological and molecular analysis excluding ex- tinct taxa}

Only one MPT of 5680 steps was obtained (result not shown). The unrooted tree is similar to that obtained with all the molecular data (Fig. 2A). The main difference is the position of Emydidae + Platysternon megacephalum Gray 1831 as the adjacent group to Chelydridae + Chelonioidea. As in the unrooted tree of all the molecular data, Trionychia is the adjacent group of Pleurodira (also retrieved in most molecular analysis), and Kinosternoidea is the adjacent group of Pleurodira+Trionychia.

\section{Morphological analysis excluding extinct taxa}

The cladistic analysis of the morphological data excluding all extinct taxa resulted in 35 MPTs of 185 steps. The strict consensus of all trees is shown as an unrooted phylogenetic tree (Fig. 3) to facilitate com-

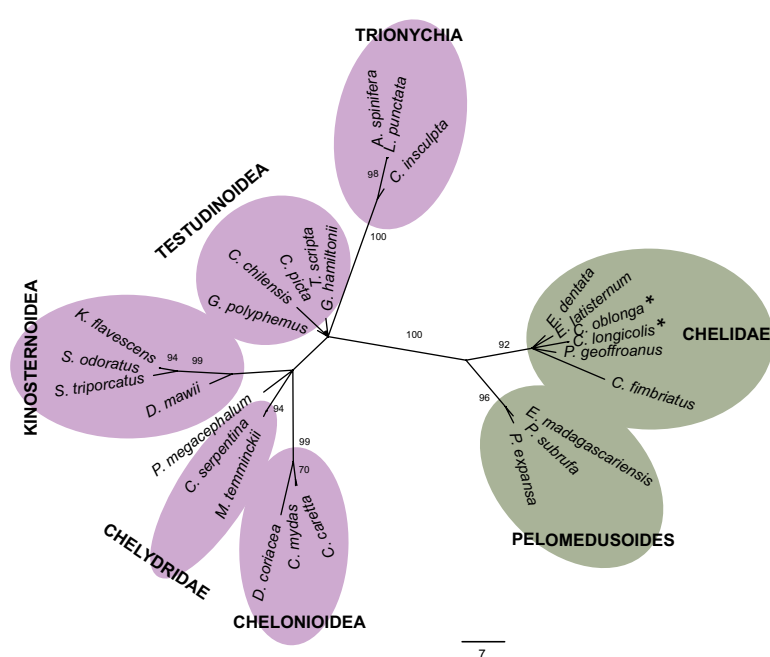

Fig. 3. Morphological phylogenetic analysis of extant turtles. Strict consensus of 35 MPTs of 185 steps each. * The monophyletic group formed by Chelodina longicollis and C. oblonga supported by a Jackknife GC value of 83 . Jackknife values below 70 are not shown. Pink: Cryptodiran clades. Green: Pleurodiran clades.

parisons with the other partitioned analyses of molecular data. In the strict consensus Trionychia is not the adjacent group to Pleurodira but it forms part of a polytomy together with some testudinids, geoemydids, and emydids (labeled as Testudinoidea in Figure 3 ). However, the general topology of this consensus tree resembles more to the unrooted topology of the molecular analyses than to the results of the morphological dataset when extinct taxa were included (Fig. 1). All the external branches are moderately to well supported (Jackknife values $>70 \%$ ), but as in the other analyses, the Jackknife values of internal branches are low (except for the one separating Pleurodira from Cryptodira).

\section{Total evidence analysis}

As a result of the total evidence analysis, 12960 most parsimonious trees (MPTs) of 5972 steps were found. A strict consensus of all trees was calculated and is shown in Fig. 4 (common synapomorphies are listed in SI 9). Although the main clades of cryptodiran turtles were recovered as monophyletic, the monophyly of Cryptodira was rejected because Pleurodira is deeply nested within this group. Cheloniidae and related taxa are sister group of the remaining crown group taxa. 
A

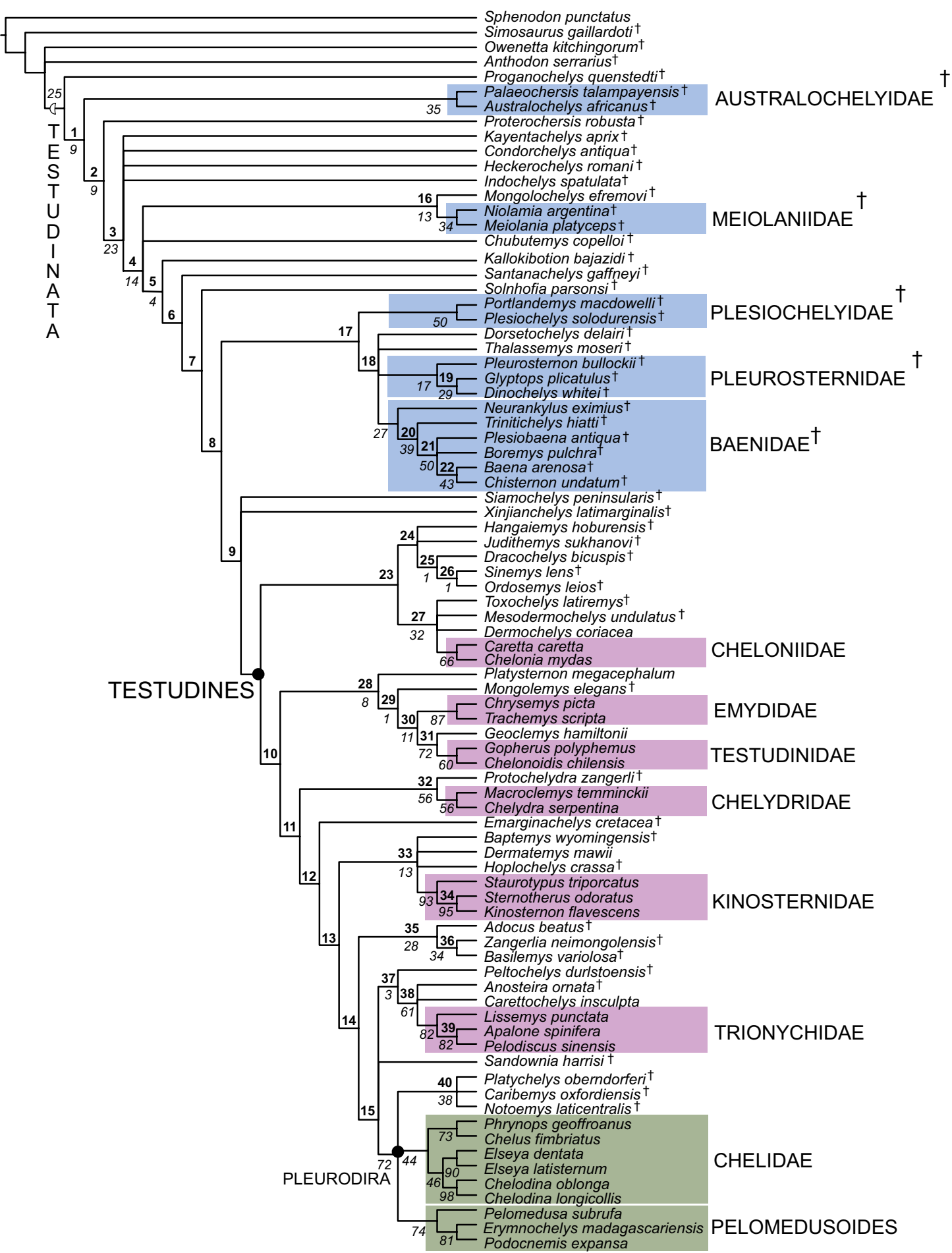

Fig. 4. Total evidence phylogenetic analysis. Strict consensus tree of 12960 MPTs of 5972 steps each. Numbers represent GC Jackknife values. Numbers in bold above the nodes represent node number. Numbers in italics under the nodes represent GC Jackknife values. Light blue: extinct recognized clades. Pink: Cryptodiran clades. Green: Pleurodiran clades. 
Pleurodira is placed as the sister group of Trionychia in this analysis, although this clade is not supported under Jackknife resampling. Most nodes have low jackknife values, except those of the clades Kinosternidae, Trionychidae, Pelomedusidae, and the genera Elseya Gray 1867 and Chelodina Fitzinger 1826 (Fig. 4).

The most unorthodox result of these topologies is the paraphyly of Cryptodira due to the position of Pleurodira as the sister group of Trionychia. In order to test the robustness of this result, a constrained analysis was performed forcing the monophyly of Cryptodira. This analysis resulted in approximately 27,000 MPTs of 5977 steps, which are 5 steps longer than MPTs of the unconstrained analysis. A total evidence analysis using Joyce's (2007) morphological dataset retrieved the same result as the total evidence analysis shown in the present paper, Pleurodira being nested inside Cryptodira and Trionychia being its sister group.

The result obtained from the present total evidence analysis, is an unorthodox result that, as it will be explained bellow, could be caused by the combination of two main factors: the strong phylogenetic signal of the molecular data that relates Pleurodira with Trionychia and the strong signal of the extinct turtles that roots Testudines in the clade leading to Chelonioidea.

\section{Discussion}

\section{Position of Pleurodira and the rooting problem}

As was mentioned above, comparison between the total evidence analysis and the morphological analysis

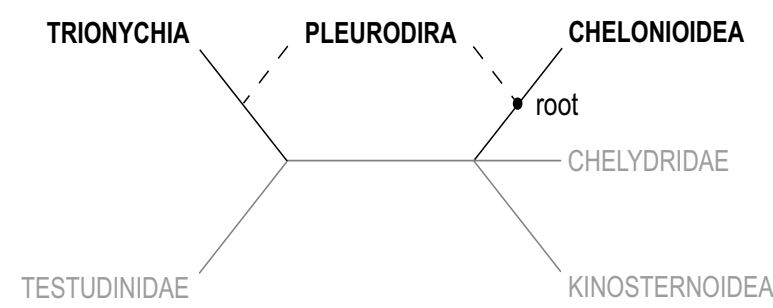

Fig. 5. Simplified unrooted tree of cryptodiran turtles (based on morphological analysis of extant taxa) showing the alternative position of Pleurodira: Morphological analysis shows that Chelonioidea and Pleurodira are the successive sister groups of remaining Cryptodira, while total evidence analysis suggests Trionychia and Pleurodira as successive sister groups. The root of Testudines is placed in the branch leading to Chelonioidea in the total evidence analysis and in the morphological analysis including fossils.
(Fig. 1,4) indicates that the main difference is the position of Pleurodira, which is independent from the rooting of the tree. Illustrated in a simplified unrooted tree the interrelationships among the major clades of cryptodiran turtles, the two alternative positions of Pleurodira are shown (Fig. 5; SI 10).

Direct comparisons with previous phylogenetic studies based on molecular data of crown-turtles are hampered by the rooting of the phylogenetic trees of most molecular studies published to date. The main difference between the previous analyses and the analyses presented here is the location of the root. If the results of previous inclusive phylogenetic analyses of turtle evolution are viewed as unrooted trees (e.g. cytochrome b of Shaffer et al., 1997; RAG-1 of Krenz et al., 2005; R35 of Fujita et al., 2004 and of Near et al., 2005; Thomson and Shaffer, 2010), Pleurodira is depicted as the adjacent group to Trionychia (Trionychidae + Carettochelyidae) in all the mentioned cases, as in the analyses reported here. Most molecular studies have assumed a priori the monophyly of Pleurodira and Cryptodira and rooted the phylogenetic trees at the branch that joins these two groups (e.g. Shaffer et al., 1997; Fujita et al., 2004; Thomson and Shaffer, 2010; and possibly Near et al., 2005). In many of these studies, therefore, Trionychia is depicted as the most basal group of Cryptodira, followed by Kinosternoidea, and Chelonioidea and Chelydridae are depicted as the most derived cryptodirans.

An exception to this a priori assumption of monophyly is the parsimony analysis of Krenz et al. (2005) of the RAG-1. These authors tested the monophyly of Pleurodira and Cryptodira adding two outgroup species [(Gallus gallus (Linneaus 1758) and Alligator mississippiensis (Daudin 1802)]. In the parsimony analysis, the root was located in the branch that leads to Trionychia, which was the longest branch of the tree, and therefore Cryptodira was depicted as paraphyletic, but again placing Trionychia as the most basal clade. In the maximum likelihood analysis the authors recovered Cryptodira as monophyletic arguing that the non-monophyletic Cryptodira resulted from a methodological issue under maximum parsimony, however, the support for this clade in the maximum likelihood approach was weak (56\%).

Placing the position of the root for crown-group turtles is highly problematic since most sequences show short internal branches separating the major groups and relatively long terminal branches. Furthermore, all extant outgroups (e.g. lepidosaurs, archosaurs, mammals) 
are highly divergent and separated from crown turtles by an extremely long branch. As pointed out by Wheeler (1990) and Shavit et al. (2007), finding the location of the root in these cases is highly problematic and in some cases the outgroup taxa may attach to the longest branches of the ingroup. This is the case, for example, of the preliminary analyses performed during this study where up to four taxa were included as outgroup (Sphenodon punctatus, Gallus gallus, Alligator mississippiensis, and Monodelphis domestica Wagner, 1842) and analyzed under maximum parsimony (MP). In the 55\% of the analyses (mainly cytochrome b, Rag-1, and concatenated), the outgroup taxa fixed to the long branch of Carettochelys insculpta (Trionychia), while only the $34 \%$ of the analyses fixed the outgroup taxa between Cryptodira and Pleurodira (12S RNA and 16S RNA). For more information see SI 10 and 11. Similar longstanding phylogenetic problems are also present in other groups of amniotes that result from a rapid evolutionary radiation, such as the debated interrelationships among major modern lineages of insects, mammals, birds, and angiosperms (Cooper and Penny, 1997; Springer et al., 2003; Soltis et al., 2004; Whitfield and Kjer, 2008).

In this regard, morphology, and particularly the morphological information present in extinct taxa, can provide useful information to resolve the position of the root of the ingroup (see above). In the total evidence analysis the position of the root of the crown group Testudines is placed close to Cheloniidae. The addition of the molecular sequences moves Pleurodira as the sister group of the derived cryptodiran clade Trionychia but does not alter the position of the root that is robustly supported by the morphological information given by the extinct species.

Short and not well supported internal branches and long external branches in molecular analyses: Rapid radiation or lack of information?

A common feature shared by all the molecular analyses presented here is the weak Jackknife support $(<70 \%)$ of the internal branches connecting the major clades of cryptodiran turtles (Figure 2). This is not unique to the present analysis, but for almost all the molecular analyses published until now (Shaffer et al., 1997; Fujita et al., 2004; Krenz et al., 2005). The only exception seems to be the supermatrix analysis of Thomson and Shaffer (2010), where some internal nodes are recovered with moderate to good support values $(>70 \%)$. Another feature of the molecular analyses presented here and in previous ones (Shaffer et al., 1997; Fujita et al., 2004; Krenz et al., 2005) is that the internal branches (internodes) are shorter than the branches leading to the terminal branches. Despite of the low support among cryptodiran lineages, the branches leading to the major clades are usually well supported. The available evidence suggests that more data (morphological and molecular) is needed to comprehend whether this low or inexistent support and the short internal branches and the long external branches are due to lack of data (soft polytomy, Coddington and Scharff, 1996) due to a rapid radiation of the crown-group, as it was proposed before by Shaffer et al. (1997) and Danilov and Parham (2006). A rapid radiation scenario is also supported by the fossil record. Under the phylogenetic hypothesis accepted here (Fig. 1) the first representatives of crown-group Testudines appear between the Middle and Late Jurassic (see also Danilov and Parham, 2008) and soon after the diversification of Testudines is evident in the fossil record by the origin of many new clades.

\section{Importance of fossils}

The comparison between the morphological analysis with extinct taxa and the total evidence analysis suggest Chelonioidea and Trionychia as the most basal clade of Cryptodira, respectively. If we contrast both hypotheses with the fossil record, it is remarkable that the minimum age for the clade Testudines is Upper Jurassic and it is given by the stem trionychian Yehguia tatsuensis (Yeh 1963) from Sichuan Province (China) (Danilov and Parham, 2006). On the other hand, the oldest records of total-group Chelonioidea are much younger, dating from the Lower Cretaceous (Bouliachelys Kear and Lee 2006, Notochelone Lydekker 1889, and possible Santanachelys Hirayama 1998; in Kear and Lee, 2006). Although the paleontological record seems to be more consistent with the total evidence hypothesis, accounting for the nature of the fossil record, new findings could change this statement. The fossils are not only useful to date the minimum age of clade origin, but bear unique combination of characters that can help to test the relationships among extant groups (Gauthier et al., 1988; Donoghue et al., 1989). The importance of extinct turtles for testing the relationships of the major groups of crown turtles is highlighted when the result of the analysis of the morphological dataset including all available taxa (both extinct and extant; see Fig. 1) 
is compared with that of the same morphological dataset but including only extant taxa (Fig. 3). The addition of extinct taxa is important to assess the internal relationships of Cryptodira, resolving several polytomies including the one that clusters Trionychia with all the members of Testudinoidea (Testudinidae + Emydidae + Geoemydidae) and the one that joins Kinosternoidea, Chelonioidea, and Chelydridae. Furthermore, the inclusion of extinct taxa provides the root of the crown group in both the morphological analyses including extinct taxa and the total evidence. The extinct taxa place the root of the crown group between Cryptodira and Pleurodira, being Cheloniidae and its sister clade ('sinemyds' and 'macrobaenids') the sister group of all remaining cryptodiran turtles (Figs 1,4). The inclusion of only one basal turtle (Proganochelys quenstedti) in the morphological analysis of extant taxa roots the crown group in the branch between Cryptodira and Pleurodira. Moreover, when only one extinct taxon of the crown group (e.g. Ordosemys leios Brinkman and Peng 1993) is added, the root of the crown Cryptodira is placed in the branch leading to Cheloniidae. The maintenance of the position of the root with the addition of more extinct taxa and even in the total evidence analysis indicates its robustness.

\section{Testing the effects of long branches}

As seen in the partitioned molecular analyses and in the concatenated molecular dataset (Fig. 2, SI 12), Trionychia (Trionychidae + Carettochelyidae) and Pleurodira (Chelidae + Pelomedusoides) are depicted as adjacent groups and the branches leading to these two groups are among the longest branches of the entire tree. In order to test whether the position of Pleurodira as the adjacent group to Trionychia could be related to the long-branch attraction problem, several analyses excluding Pleurodira, Trionychia, and Kinosternoidea were performed (see SI 12-18). The results show that the phylogenetic relationships among these clades are usually stable and their position does not change when one of the mentioned taxa is excluded from the analysis. Following the suggestions of Siddall and Whiting (1999) and Pol and Siddall (2001) these results can be interpreted as a rejection of a LBA explanation for the close position of Pleurodira and Trionychia. To further test this, the five genes were used separately in Bayesian analyses and maximum likelihood. In all the cases the topologies (SI 19) retrieved Trionychia as the adjacent group of Pleurodira.

\section{Conclusions}

The most outstanding results of this contribution are the alternative position of Pleurodira according to the different sources of data and the robustness of the root given by the morphological information of extinct taxa. A major point of disagreement between the morphological information (especially those on extinct taxa) and the molecular information (especially the cytochrome b, 12S RNA, and RAG-1) is the contradictory phylogenetic signal regarding the position of Pleurodira, which allies this group closer to Cheloniidae or Trionychia, respectively. On the other hand, the signal of the extinct taxa to root the crown-group Testudines near the clade leading to Cheloniidae is so strong that it prevails not only in the morphological tree with extinct taxa, but in the total evidence tree as well. The combination of the position of Pleurodira as the sister group of Trionychia in the molecular analyses and the strong signal of the root in crown-group Testudines results in the unorthodox result of Cryptodira being paraphyletic with Pleurodira well nested inside in the total evidence analysis. The results shown in this paper highlight promising future areas of research, both within the morphological and on the molecular fields. In addition to gathering more character data, future studies should include new extinct and extant taxa from crown-group turtles, to recover more information about the origin and diversification of crown-group Testudines, one of the most peculiar clades of living amniotes.

\section{Acknowledgements}

Thanks to M.S. de la Fuente for his support, S. Espert and S. Catalano for their help with the molecular data analyses, and D. Pol for his help with all the topics concerning cladistic methodology. E. Ruigomez (Museo Paleontológico Egidio Feruglio, Argentina), M. Reguero and J. Williams (Museo de La Plata, Argentina), A. Kramarz and J. Faivovich (Museo Argentino de Ciencias Naturales, Argentina), S. Chapman (The Natural History Museum, UK), A. Wynn and T. Hartsel (United States National Museum, USA), E.S. Gaffney and C. Mehling (American Museum of Natural History, USA), W.G. Joyce (Yale Peabody Museum, USA), E. Müller-Merz (Naturmuseum Solothurn, Switzerland), C. Meyer (MH, Switzerland), J. Müller (Humboldt-Universität zu Berlin Museum für Naturkunde, Germany), and R. Schoch (Staaliches Museum für Naturkunde Stuttgart, Germany) are thanked for the access to comparative collection. M.S. de la Fuente and D. Pol are also thanked for reading early versions of this manuscript. Special thanks are given to W.G. Joyce, M. Laurin, and an anonymous reviewer for the detailed revision that greatly improved the quality of the manuscript. This work was partially 
supported by the Richard Estes Memorial Grant, a DAAD fellowship to J.S., 'Exploración paleontológica de la Patagonia' grant of Lampadian Foundation and RIG5598 grant of University of Louisville (G.W. Rougier), PIP grant 112-200801-00795 (M.S. de la Fuente), and PICT grant 2006-1756 (D. Pol).

\section{References}

Anquetin J, Barrett PM, Jones MEH, Moore-Fay S, Evans SE. 2009. A new stem turtle from the Middle Jurassic of Scotland: new insights into the evolution and palaeoecology of basal turtles. Proceedings of the Royal Society B 276: 879886.

Brinkman D, Wu X-C. 1999. The skull of Ordosemys, an Early Cretaceous turtle from Inner Mongolia, People's Republic of China, and the interrelationships of Eucryptodira (Chelonia, Cryptodira). Paludicola 2:134-147.

Burke AC. 1989. Development of the turtle carapace: implications for the evolution of a novel Bauplan. Journal of Morphology 199: 363-378.

Coddington JA, Scharff K. 1996. Problems with 'soft' polytomies. Cladistics 12:139-145.

Cooper A, Penny D. 1997. Mass survival of birds across the Cretaceous-Tertiary boundary: molecular evidence. Science 275: 1109-1113.

Danilov IG, Parham JF. 2006. A redescription of 'Plesiochelys' tatsuensis from the Late Jurassic of China, with comments on the antiquity of the crown clade Cryptodira. Journal of Vertebrate Paleontology 26: 573-580.

Danilov IG, Parham JF. 2008. A reassessment of some poorly known turtles from the Middle Jurassic of China, with comments on the antiquity of extant turtles. Journal of Vertebrate Paleontology 28: 306-318.

deBraga M, Rieppel O. 1997. Reptile phylogeny and the interrelationships of turtles. Zoological Journal of the Linnean Society 129: 281-354.

Donoghue MJ, Doyle JA, Gauthier J, Kluge AG, Rowe T. 1989. The importance of fossils in phylogenetic reconstruction. Annual Review of Ecology and Systematics 20: 431-460.

Engstrom TN, Shaffer HB, McCord WP. 2004. Multiple dara sets, high homoplasy, and the phylogeny of softshell turtles (Testudines: Trionychidae). Systematic Biology 53: 693-710.

Fujita MK., Engstrom TN, Starkey DE, Shaffer HB. 2004. Turtle phylogeny: insights from a novel nuclear intron. Molecular Phylogenetics and Evolution 31: 1031-1040.

Gaffney ES. 1975. A phylogeny and classification of the higher category of turtles. Bulletin of the American Museum of Natural History 155: 389-436.

Gaffney ES. 1996. The postcranial morphology of Meiolania platyceps and a review of the Meiolaniidae. Bulletin of the American Museum of Natural History 229: 1-166.

Gaffney ES, Meylan PA. 1988. A phylogeny of turtles. Pp. 157219 in: Benton MJ, ed., The Phylogeny and Classification of the Tetrapods. Oxford: Clarendon Press.

Gaffney ES, Rich TH, Vickers-Rich P, Constantine A, Vacca R, Kool L. 2007. Chubutemys, a new eucryptodiran turtle from the Early Cretaceous of Argentina, and the relationships of Meiolaniidae. American Museum Novitates 3599: 1-35.
Gauthier J, Kluge AG, Rowe T. 1988. Amniote phylogeny and the importance of fossils. Cladistics 4: 105-209.

Giribet G, Wheeler WC. 1999. On gaps. Molecular Phylogenetics and Evolution 13: 132-143.

Goloboff PA, Farris JS, Källersjö M, Oxelman B, Ramírez MJ, Szumik CA. 2003. Improvements to resampling measures of group support. Cladistics 19: 324-332.

Goloboff P, Farris J, Nixon K. 2008a. TNT: Tree Analysis Using New Technology, version 1.1 (Willi Hennig Society Edition). Program and documentation available at http:// www.zmuc.dk/public/phylogeny/tnt.

Goloboff PA, Farris JS, Nixon K. 2008b. A free program for phylogenetic analysis. Cladistics 24: 774-786.

Hill RV. 2005. Integration of morphological data sets for phylogenetic analysis of Amniota: The importance of integumentary characters and increased taxonomic sampling. Systematic Biology 54: 530-547.

Hirayama R, Brinkman DB, Danilov IG. 2000. Distribution and biogeography of non-marine Cretaceous turtles. Russian Journal of Herpetology 7: 181-198.

Iverson JB, Brown RM, Akre TS, Near TJ, Le M, Thomson RC, Starkey DE. 2007. In search of the tree of life for turtles. Pp 85-106 in: Shaffer HB, FitzSimmons NN, Georges A, Rhodin AGJ, eds., Defining Turtle Diversity: Proceedings of a Workshop on Genetics, Ethics, and Taxonomy of Freshwater Turtles and Tortoises. Chelonian Research Monographs, vol. 4.

Joyce WG. 2007. Phylogenetic relationships of Mesozoic turtles. Peabody Museum Bulletin 48: 3-102.

Joyce WG, Parham JF, Gauthier JA. 2004. Developing a protocol for the conversion of rank-based taxon names to phylogenetically defined clade names, as exemplified by turtles. Journal of Paleontology 78: 989-1013.

Kear BP, Lee MYS. 2006. A primitive protostegid from Australia and early sea turtle evolution. Biology Letters 2: 116119.

Krenz JG, Naylor GJP, Shaffer HB, Janzen FJ. 2005. Molecular phylogenetics and evolution of turtles. Molecular Phylogenetics and Evolution 37: 178-191.

Lanyon SM. 1985. Detecting internal inconsistencies in distance data. Systematic Zoology 34: 397-403.

Laurin M, Reisz RR. 1995. A reevaluation of early amniote phylogeny. Zoological Journal of the Linnean Society 113: 165-223.

Lee MSY. 1993. The origin of the turtle body plan: bridging a famous morphological gap. Science 261: 1716-1720.

Lee MSY. 1995. Historical burden in systematics and the interrelationships of 'Parareptiles'. Biological Review 70: 459547.

Lee MSY. 1996. Correlated progression and the origin of turtles. Nature 379: 812-815.

Lee MSY. 1997. Pareiasuar phylogeny and the origin of turtles. Zoological Journal of the Linnean Society 120: 197-280.

Müller J. 2003. Early loss and multiple return of the lower temporal arcade in diapsid reptiles. Naturwissenschaften 90: 473-476.

Müller J. 2004. The relationships among diapsid reptiles and the influence of taxon selection. Pp. 379-408 in: Arratia G, Wilson MVH, Cloutier R, eds., Recent Advances in the Origin and Early Radiation of Vertebrates. München: Verlag Dr. Friedrich Pfeil. 
Near TJ, Meylan PA, Shaffer HB. 2005. Assessing concordance of fossil calibration points in molecular clock studies: an example using turtles. American Naturalist 165: 137-146.

Parham JF, Feldman CR, Boore JL. 2006. The complete mitochondrial genome of the enigmatic bigheaded turtle (Platysternon): description of unusual genomic features and the reconciliation of phylogenetic hypotheses based on mitochondrial and nuclear DNA. BMC Evolutionary Biology 6: 1-11.

Pol D, Siddall ME. 2001. Biases in maximum likelihood and parsimony: a simulation approach to a 10 -taxon case. Cladistics 17: 266-281.

Reisz RR, Laurin M. 1991. Owenetta and the origin of turtles. Nature 349: 324-326.

Rieppel O, deBraga M. 1996. Turtles as diapsid reptiles. $\mathrm{Na}$ ture 384: 453-455.

Rieppel O, Reisz RR. 1999. The origin and early evolution of turtles. Annual Review of Ecology and Systematics 30: 1-22.

Rougier GW, de la Fuente MS, Arcucci AB. 1995. Late Triassic turtles from South America. Science 268: 855-858.

Shaffer HB, Meylan PA, McKnight ML. 1997. Test of turtle phylogeny: molecular, morphological, and paleontological approaches. Systematic Zoology 46: 235-268.

Shavit L, Penny D, Hendy MD, Holland BR. 2007. The problem of rooting rapid radiations. Molecular Biology and Evolution 24: 2400-2411.

Siddall ME, Whiting MF. 1999. Long-branch abstractions. Cladistics 15: 9-24.

Soltis DE, Albert VA, Savolainen V, Hilu K, Qiu Y-L, Chase MW, Farris JS, Stefanovic S, Rice DW, Palmer JD, Soltis PS. 2004. Genome-scale data, angiosperm relationships, and 'ending incongruence': a cautionary tale in phylogenetics. Trends in Plant Science 9: 477-483

Springer MS, Murphy WJ, Eizirik E, O’Brien SJ. 2003. Placental mammal diversification and the Cretaceous-Tertiary boundary. Proceedings of the National Academy of Sciences 100: 1056-1061.

Sterli J. 2008. A new, nearly complete stem turtle from the Jurassic of South America with implications for turtle evolution. Biology Letters 4: 286-289.

Sterli J, de la Fuente MS, Rougier GW. 2007. Anatomy and relationships of Palaeochersis talampayensis, a Late Triassic turtle from Argentina. Palaeontographica Abteilung A 281: 1-61.

Thompson JD, Gibson TJ, Plewniak F, Jeanmougin F, Higgins DG. 1997. The ClustalX windows interface: flexible strategies for multiple sequence alignment aided by quality analysis tools. Nucleic Acids Research 25: 4876-4882.

Thomson RC, Shaffer HB. 2010. Sparse supermatrices for phylogenetic inference: Taxonomy, alignment, rogue taxa, and the phylogeny of living turtles. Systematic Biology 59: 42-58.

Werneburg I, Sánchez-Villagra MR. 2009. Timing of organogenesis support basal position of turtles in the amniote tree of life. BMC Evolutionary Biology 9: 82-91.

Wheeler WC. 1990. Nucleic acid sequence phylogeny and random outgroups. Cladistics 6: 363-367.

Whitfield JB, Kjer KM. 2008. Ancient Rapid Radiations of Insects: Challenges for Phylogenetic Analysis. Annual Review of Entomology 53: 449-472.

Wilkinson M, McInerney JO, Hirt RP, Foster PG, Embley TM. 2007. Of clades and clans: terms for phylogenetic relationships in unrooted trees. Trends in Ecology and Evolution 22: $114-115$.

Received: 29 June 2010

Revised and accepted: 23 August 2010

Published online: 24 September 2010

Editor: J.W. Arntzen 


\section{On-line supplementary information (SI)}

SI 1: Character list and changes to Sterli's (2008) matrix.

SI 2: Morphological data set.

SI 3: GenBank accession numbers of all the sequences (12S RNA, 16S RNA, cytochrome b, RAG-1, and intron fingerprint protein R35).

SI 4: 12S RNA data set.

SI 5: 16S RNA data set.

SI 6: Cytochrome b data set.

SI 7: RAG-1 data set.

SI 8: Intron of the fingerprint protein R35 data set.

SI 9: List of synapomorphies of total evidence analysis, and morphological analysis with extinct and extant taxa.

SI 10: Table showing the two main alternative position of Pleurodira and the different position of the root in crown-group turtles. A) Simplified unrooted topology of morphological data with extinct and extant taxa. B) Simplified unrooted topology of molecular data. Circles denote rooting.

SI 11: Table showing the summarized results of the different analyses performed including outgroup taxa to molecular data. These analyses were exploratory and they were performed under maximum parsimony (MP).

SI 12: Fig. 3 at species level. A) Molecular analysis of the 5 sequences (12S RNA, 16S RNA, cytochrome b, RAG-1, and intron of the fingerprint protein R35). Only one MPT of 5483 steps were recovered. B) Molecular analysis of cytochrome b sequence. Only one MPT of 2061 steps was recovered. C) Molecular analysis of 12S RNA sequence. Strict consensus of two MPTs of 644 steps each. D) Molecular analysis of the RAG-1 sequence. Only one MPT of 927 steps was recovered. E) Molecular analysis of 16S RNA sequence. Only one MPT was found of 838 steps. F) Molecular analysis of the intron of the fingerprint protein 35. Strict consensus of two MPTs of 972 steps each.

SI 13: Unrooted trees showing the phylogenetic relationships of turtles using 12S RNA sequence.

A) Without Pleurodira. B) Without Trionychia.

C) Without Trionychia and Pleurodira. D)

Without Trionychia and Kinosternoidea.
SI 14: Unrooted trees showing the phylogenetic relationships of turtles using 16S RNA sequence.

A) Without Pleurodira. B) Without Trionychia.

C) Without Trionychia and Pleurodira. D) Without Trionychia and Kinosternoidea.

SI 15: Unrooted trees showing the phylogenetic relationships of turtles using cytochrome $b$ sequence. A) Without Pleurodira. B) Without Trionychia. C) Without Trionychia and Pleurodira. D) Without Trionychia and Kinosternoidea.

SI 16: Unrooted trees showing the phylogenetic relationships of turtles using RAG-1 sequence. A) Without Pleurodira. B) Without Trionychia. C) Without Trionychia and Pleurodira. D) Without Trionychia and Kinosternoidea.

SI 17: Unrooted trees showing the phylogenetic relationships of turtles using intron fingerprint protein R35 sequence. A) Without Pleurodira. B) Without Trionychia. C) Without Trionychia and Pleurodira. D) Without Trionychia and Kinosternoidea.

SI 18: Unrooted trees showing the phylogenetic relationships of turtles using all the molecular data (12S RNA, 16S RNA, cytochrome b, RAG-1, and intron fingerprint protein R35 sequences). A) Without Pleurodira. B) Without Trionychia. C) Without Trionychia and Pleurodira. D) Without Trionychia and Kinosternoidea.

SI 19: Bayesian analysis results. A) Cytochrome b sequence. B) 12S RNA sequence. C) RAG-1 sequence. D) $16 \mathrm{~S}$ RNA sequence. E) Intron of the fingerprint protein 35 sequence. Shown values: posterior probabilities (asterisks denote 100\%). Values below $80 \%$ are not shown. Bayesian analyses were performed using MrBayes (Huelsenbeck and Ronquist, 2001. MrBayes: Bayesian inference of phylogeny. Bioinformatics 17:754-755). Two analyses were run simultaneously and they were initiated with random trees. The analyses run for $1,000,000$ generations and the Markov chains were sampled every 100 generations. MrModeltest v2 (Nylander, 2004. MrModeltest v2. Evolutionary Biology Centre, Uppsala University, Uppsala) found the GTR + G model is more accurate for 12S RNA, 16S RNA, and intron R35 genes, and GTR + I + G model for cytochrome $b$ and RAG-1 (the same as in ML analyses). 